\title{
A Mathematical Model for Simultaneous Spatio-Temporal Dynamics of Calcium and Inositol 1,4,5-Trisphosphate in Madin-Darby Canine Kidney Epithelial Cells
}

\author{
T. Roose ${ }^{\mathrm{a}, \mathrm{b}, *}$, S. J. Chapman ${ }^{\mathrm{a}}$, P. K. Maini ${ }^{\mathrm{b}}$ \\ ${ }^{a}$ Centre for Industrial and Applied Mathematics, Mathematical Institute, \\ Oxford University, 24-29 St. Giles,' Oxford OX1 3LB, UK \\ ${ }^{b}$ Centre for Mathematical Biology, Mathematical Institute, Oxford University, \\ 24-29 St. Giles,' Oxford OX1 3LB, UK
}

Received: 19 May 2005 / Accepted: 30 November 2005 / Published online: 26 July 2006 (C) Society for Mathematical Biology 2006

\begin{abstract}
The landmark paper by Hirose et al. (Hirose, K., Kadowaki, S., Tanabe, M., Takeshima, H., Iino, M., Science 284:1527-1530, 1999) presented experimental investigations to show that not only can calcium upregulate $\mathrm{IP}_{3}$, but that it can also have an inhibitory effect on $\mathrm{IP}_{3}$. In this paper, we present a preliminary model, which is consistent with these experiments. This model includes positive and negative feedback between calcium and $\mathrm{IP}_{3}$ and is able to reproduce more precisely the data presented in Hirose et al. (Hirose, K., Kadowaki, S., Tanabe, M., Takeshima, H., Iino, M., Science 284:1527-1530, 1999). In the second part of the paper, the intracellular and intercellular calcium movement in Madin-Darby canine kidney epithelial cells is investigated. With the aid of the model we are able to identify the aspects of $\mathrm{IP}_{3}$ and calcium signalling, which should be studied further experimentally before refining the model.
\end{abstract}

Keywords Mathematical model C Calcium dynamics · Inositol 1,4,5-trisphosphate

Abbreviations $\mathrm{IP}_{3}$, inositol 1,4,5-trisphosphate; ER, endo(sarco)plasmic reticulum; $\mathrm{IP}_{3} \mathrm{R}$, inositol 1,4,5-trisphosphate receptor; ATP, adenosine triphosphate; $\mathrm{GFP}$, green fluorescent protein.

\section{Introduction}

Out of $1.4 \mathrm{~kg}$ of calcium in a human body most is located in the skeleton and teeth, and only $10 \mathrm{~g}$ is located elsewhere (Carafoli, 2003). This $10 \mathrm{~g}$ of calcium is responsible for a wide variety of signalling behaviour observed in soft tissues (Berridge

*Corresponding author.

E-mail address: roose@maths.ox.ac.uk (T. Roose). 


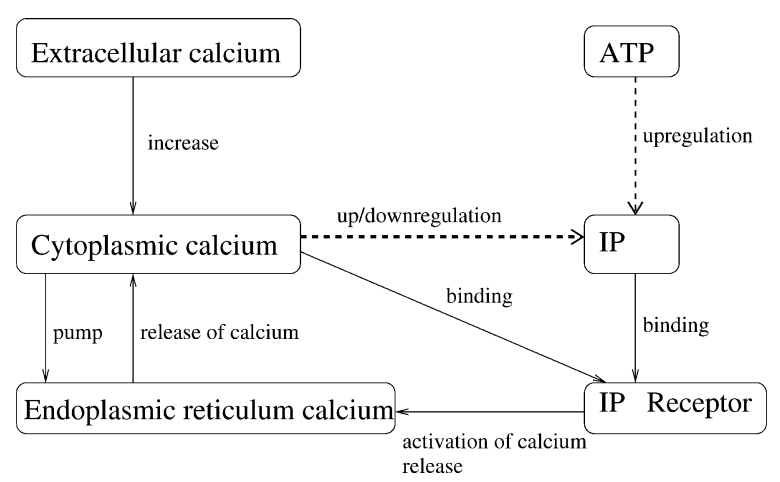

Fig. 1 Schematic representation of the calcium and $\mathrm{IP}_{3}$ interaction. Solid lines show the 'classical' dynamics, which combined with the dashed lines, describe the model in this paper.

et al., 2000). Therefore, calcium signalling is an area of intense research in biology, molecular biology and other related disciplines. Most of the research into the calcium signalling in soft tissues has been accompanied by active mathematical modelling. Thus, there are many mathematical models for this phenomenon present in the literature (reviewed by Schuster et al., 2002; Sneyd, 2005). Classical intracellular and intercellular calcium signalling models consider calcium to be present inside cells in at least two different pools: Calcium in the cytoplasm and calcium bound to endo(sarco)plasmic reticulum (ER/SR). In many cells the movement of calcium between the two pools occurs via inositol 1,4,5-trisphosphate receptors $\left(\mathrm{IP}_{3} \mathrm{R}\right)$ on ER. These receptors can be activated either by calcium in the cytoplasm or by inositol 1,4,5-trisphosphate $\left(\mathrm{IP}_{3}\right)$ itself (see Fig. 1 for schematic representation). In most of the existing models $\mathrm{IP}_{3}$ is one of the crucial factors determining the behaviour of calcium. However, unlike calcium, for which many experimental staining techniques are available, the techniques for visualising the spatio-temporal dynamics of $\mathrm{IP}_{3}$ have been missing until very recently. Hirose et al. (1999) provided one of the first spatio-temporal measurements of $\mathrm{IP}_{3}$ dynamics inside cells using a construct including green fluorescent protein (GFP) observed with a confocal laser microscope. All quantities Hirose et al. (1999) measured were in the units of the relative increase in fluorescence intensity with respect to basal values of fluorescence in cells in response to various stimuli, i.e. they measured $\Delta F / F_{0}$, where $F_{0}$ is the basal level of fluorescence in the cell and $\Delta F$ is the increase in the fluorescence due to stimulation. Therefore, $\Delta F / F_{0}=0$ does not imply that the concentration of $\mathrm{IP}_{3}$ is zero, rather that it is equal to the steady unstimulated level of $\mathrm{IP}_{3}$. Another important point concerning the fluorescence detection technique used is that the increase in fluorescence detected is not necessarily linearly proportional to the increase in the concentration of tagged protein, in our case $\mathrm{IP}_{3}$, as suggested by Fig. 2D in Hirose et al. (1999). Nevertheless, since all the numerical values for increase in fluorescence intensity in the main experimental results, Figs. 3 and 4, in Hirose et al. (1999) are in the 'linear range' of 0-0.2 of a 'calibration curve' on Fig. 2D, we will approximately correlate that to the increase in concentration of $\mathrm{IP}_{3}$ using $\Delta\left[\mathrm{IP}_{3}\right] \approx\left(\Delta F / F_{0}\right) \times 22 \mu \mathrm{M}$. However, one has to bear in mind that the fluorescence intensity can and does vary between experiments 


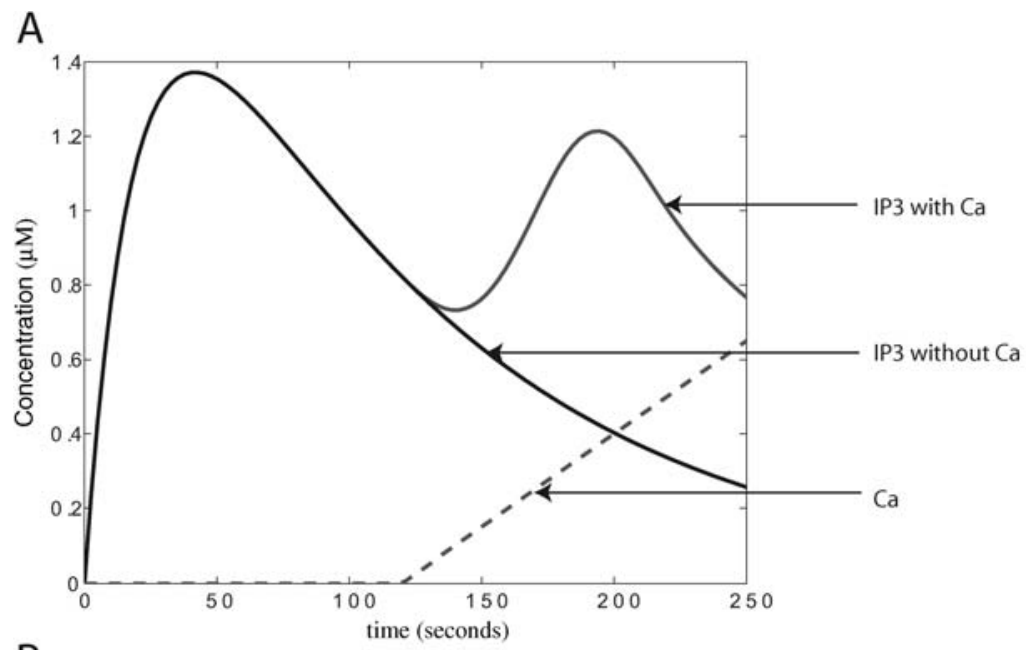

B

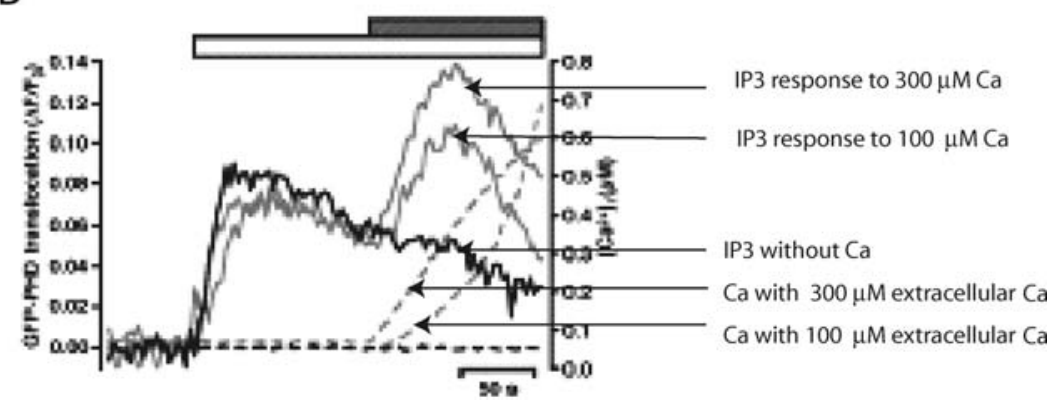

Fig. 2 (A) Upregulation of $\mathrm{IP}_{3}$ by application of $3 \mu \mathrm{M}$ of ATP only (solid line) and application of $3 \mu \mathrm{M}$ of ATP at time $t=0$ and $100 \mu \mathrm{M}$ of extracellular calcium at time $t=120 \mathrm{~s}$ (IP dashed line, $\mathrm{Ca}^{2+}$ dotted-dashed line). Calculations were done using Matlab 6.5 Release 13 and Eqs. (1) and (2). (B) Experimental data reprinted with permission from Hirose et al. (1999) (Copyright 1999, AAAS). The white bar represents the application of $3 \mu \mathrm{M}$ of ATP. The grey bar corresponds to extracellular calcium application; the response in intracellular $\mathrm{Ca}^{2+}$ concentration due to application of 0,100 and $300 \mu \mathrm{M} \mathrm{Ca}^{2+}$ extracellular $\mathrm{Ca}^{2+}$ is shown in dashed lines that are labelled accordingly. The corresponding $\mathrm{IP}_{3}$ response is shown with similar labelling.

and therefore such quantitative conversions into molar concentrations might not be very accurate.

In this paper, we develop a preliminary model that can reproduce the experimental behaviour observed in Figs. 3 and 4 of Hirose et al. (1999). Using this model we predict the behaviour of the system on a longer timescale than that observed in those experiments. The reminder of the paper is organised as follows. In Section 2.1, we discuss in detail the experimental results of Hirose et al. (1999) that most influence our new model. In Section 2.2, we discuss the previous models of calcium and $\mathrm{IP}_{3}$ signalling and point out why they are unable to reproduce these results, and therefore why a new model is needed. In Section 3, we extend the model to show more complex temporal evolution of the calcium and $\mathrm{IP}_{3}$ signalling and we also discuss the linear stability of the steady states. In Section 4, we describe the 

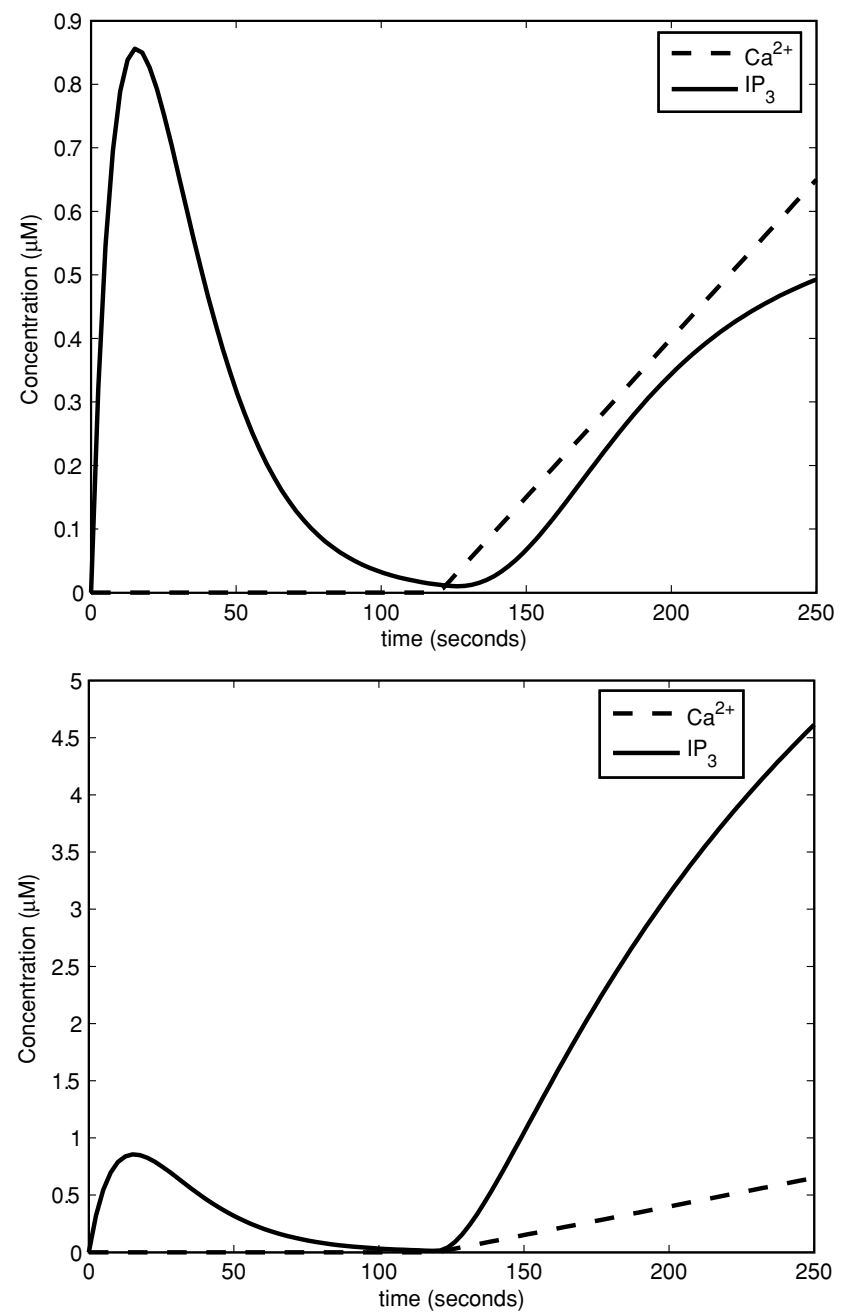

Fig. 3 Results using the Höfer et al. (2002) (top panel) and Meyer and Stryer (1991) (bottom panel) feedback terms. (1) The figure for Höfer et al. (2002) was calculated using $\mathrm{d} p / \mathrm{d} t=$ $0.05 \mu \mathrm{M} \mathrm{s}^{-1} c /(0.3 \mu \mathrm{M}+c)-0.009 \mathrm{~s}^{-1} p$ where $c$ is given by the experimental observations of Hirose et al. (1999) (our Eq. (2)), and (2) the figure for Meyer and Stryer (1991) was calculated in a similar way using $\mathrm{d} p / \mathrm{d} t=1 \mu \mathrm{M} \mathrm{s}^{-1} c /(1 \mu \mathrm{M}+c)-0.009 \mathrm{~s}^{-1} p$. Calculations show the corresponding $\mathrm{IP}_{3}$ dynamics in response to linearly increasing calcium concentration (dashed lines) as observed in the Hirose et al. (1999) experiment.

model for intracellular spatial dynamics of calcium and $\mathrm{IP}_{3}$ signalling that results from the dynamics developed in the previous sections. This model is then extended in Section 5 to intercellular spatio-temporal dynamics of calcium and $\mathrm{IP}_{3}$ in $\mathrm{MDCK}$ epithelial cells. Finally, in Section 6 we present the conclusions of our study and point out some possible further avenues of investigation. 
A
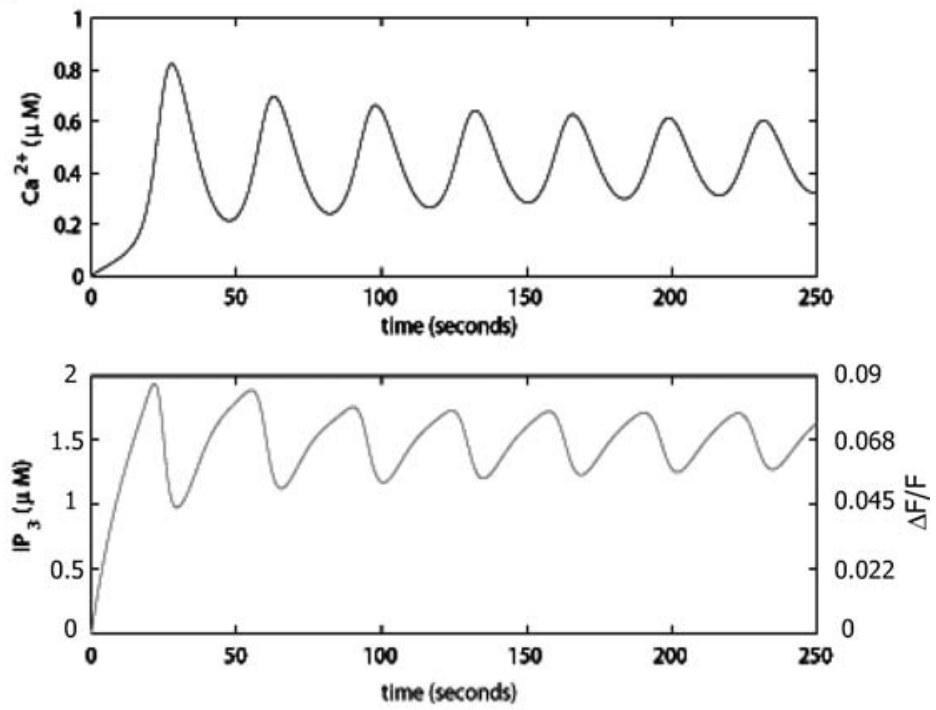

B

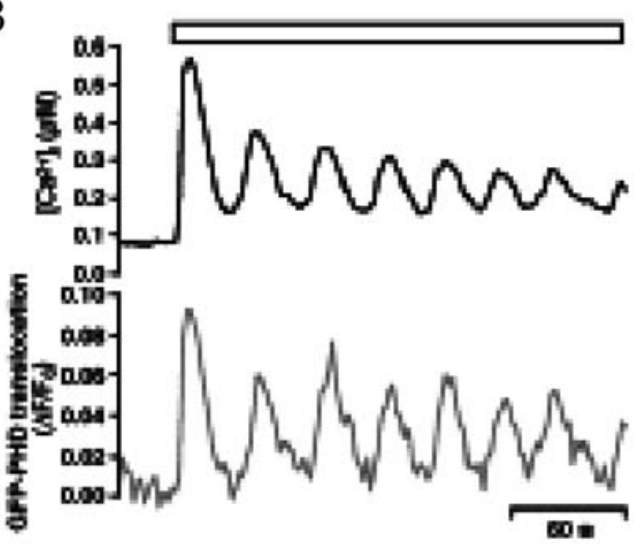

Fig. 4 (A) Simultaneous oscillations in calcium and $\mathrm{IP}_{3}$ concentration as a result of stimulation by ATP (Eqs. (4)-(7)). The initial condition is the positive steady state of the system in the absence of ATP stimulation, $c(0)=2.1 \times 10^{-3} \mu \mathrm{M}, p(0)=5.69 \times 10^{-4} \mu \mathrm{M}$ and $a(0)=3 \mu \mathrm{M}$. Numerical solution was obtained using the Matlab 6.5 Release 13 ode15s solver. (B) Experimental measurements, reprinted with permission from Hiorse et al. (1999) (Copyright 1999, AAAS), showing simultaneous oscillations in $\mathrm{Ca}^{2+}$ and $\mathrm{IP}_{3}$ as a result of ATP stimulation.

\section{2. $\mathrm{IP}_{3}$ upregulation by $\mathrm{ATP}$ and $\mathrm{Ca}^{2+}$}

\subsection{Summary of experimental results}

Hirose et al. (1999) depleted all the internal calcium stores of canine kidney epithelial cells using ionomycin in order to investigate what effect the application 
of adenosine triphosphate (ATP) and calcium have on the concentration of $\mathrm{IP}_{3}$ within the cells. ATP is a nucleotide important for energy transfer within cells, and therefore the question arises as to whether it influences $\mathrm{IP}_{3}$ directly or via its joint effect with $\mathrm{Ca}^{2+}$. The experimental evidence of Hirose et al. (1999) (see reproduction of results in Fig. $2 \mathrm{~B}^{1}$ ) suggests that ATP application activates the phosholipase $\mathrm{C}$ (PLC) in a cell membrane and that in turn produces $\mathrm{IP}_{3}$. The data from Hirose et al. (1999) also suggest that such $\mathrm{IP}_{3}$ production is independent of calcium, and that the stimulatory effect of ATP decreases in time. This might be due to several mechanisms such as ATP diffusion away from the cell, decrease of cell surface receptor activity, or other intracellular ATP modification events. In Hirose et al. (1999), there is no evidence as to which one of these mechanisms might dominate.

Following the activation of cell $\mathrm{IP}_{3}$ production by ATP application, Hirose et al. (1999) applied excess extracellular calcium and observed the level of calcium inside the cells go up (dashed lines on Hirose et al. (1999) Fig. 3B; see the reproduction in Fig. 2B of this ${ }^{2}$ paper). This treatment resulted in an initial increase in $\mathrm{IP}_{3}$ levels. However, concentrations of cytosolic calcium above a threshold level of about $0.2-0.6 \mu \mathrm{M}$ had an inhibitory effect on $\mathrm{IP}_{3}$ levels (see also Fig. $3 \mathrm{C}$ of Hirose et al., 1999).

There is an open question as to whether calcium alone, in the absence of ATP, would be able to give the upregulatory response in $\mathrm{IP}_{3}$. The experimental evidence from Hirose et al. (1999) does not give a clear answer to this as this experiment was not conducted in their paper. A partial answer comes from another paper where the same group of authors used a different cell line, Purkinje cells, and observed that calcium alone was able to give the response in $\mathrm{IP}_{3}$ Okubo et al. (2001). Thus, both ATP and $\mathrm{Ca}^{2+}$ independent of each other appear to be able to upregulate $\mathrm{IP}_{3}$ production.

\subsection{Mathematical model}

There have been many previous attempts to model $\mathrm{Ca}^{2+}$ and $\mathrm{IP}_{3}$ interaction. However, since these were all done to explain different phenomena they are unable to completely capture the experimental results of Hirose et al. (1999). For example, Atri et al. (1993) very successfully explain how periodic oscillations and spiral waves arise in the Xenopus oocytes in response to constant $\mathrm{IP}_{3}$ levels. Papers by Sneyd et al. (1995, 1998b) explain how intercellular calcium waves propagate in the airway epithelial multicell layer when one cell is mechanically stimulated. Meyer and Stryer $(1988,1991)$ explain how periodic calcium spiking can occur in the presence of constant receptor stimulation. The model of Höfer et al. (2002) identifies that multiple steps might be involved in regenerative calcium signalling in astrocytes. Whilst all the papers mentioned above deal very successfully with the specific questions that they ask, none of these models is directly applicable for analysing the data of Hirose et al. (1999). Therefore, we will take into account all

\footnotetext{
${ }^{1}$ Reprinted with permission from Hirose et al. (1999) (Copyright 1999, AAAS).

${ }^{2}$ From now on we will reference the reproduction of Hirose et al. (1999) Fig. 3B as Fig. 2B of this paper.
} 
those previous studies and use them as a foundation on which to build a model to explain the data of Hirose et al. (1999). We first briefly discuss which aspects of previous models are not consistent with the aforementioned phenomena. Firstly, most of the previous models, whilst predicting calcium oscillations, do not predict $\mathrm{IP}_{3}$ oscillations since there is no feedback from calcium dynamics to $\mathrm{IP}_{3}$ dynamics Atri et al. (1993; Sneyd et al., 1995, 1998b). Secondly, the models that do include feedback of calcium into $\mathrm{IP}_{3}$ (Meyer and Stryer, 1988, 1991; Höfer et al., 2002) only include positive feedback. However, the experiments of Hirose et al. (1999) indicate that calcium might have a positive feedback on $\mathrm{IP}_{3}$ production at low concentrations, but a negative feedback at high concentrations. Although this is not the only way to interpret the data presented by Hirose et al. (1999) it is the simplest, and we proceed with this assumption in the absence of further experimental evidence. In the conclusion, we discuss some alternative explanations of the data, and the experiments that would give us a better insight into the interplay between calcium and $\mathrm{IP}_{3}$ in MDCK epithelial cells. In addition, the models by Meyer and Stryer (1988, 1991); Höfer et al. (2002), whilst predicting simultaneous oscillations of calcium and $\mathrm{IP}_{3}$, gave values for the $\mathrm{IP}_{3}$ steady state which are higher than those estimated from experiments (Meyer and Stryer, 1988, 1991; Höfer et al., 2002; Fink et al., 1999; Luzzi et al., 2000).

As well as the minimal models mentioned above, there are also large-scale bioinformatics models that consider more than 100 chemical species in the biochemical signalling network of $\mathrm{IP}_{3}$ and calcium (Mishra and Bhalla, 2002). While such models include more biological realism they are difficult to parametrise, and mathematical techniques to analyse them fully remain to be developed so that most studies are computational. It is therefore not clear how sensitive such models are to changes in parameter values and how representative a particular computation is of the overall behaviour of the model. Therefore, in the current paper we aim to derive a minimal model that can mimic the experiments of Hirose et al. (1999) and that is consistent with experimentally measured steady-state values of the chemical messengers involved.

Based on the experimental description in Section 2.1 we choose to model the ATP effect using a quantity $a$ to be a measure of ATP efficacy, and we take $a$ to be exponentially decaying in time. We will choose the decay parameter so that it will give a good qualitative agreement with the experimental results of Hirose et al. (1999). Mathematically we model this behaviour through the differential equations ${ }^{3}$

$$
\frac{\partial a}{\partial t}=-\alpha_{1} a, \quad \frac{\partial p}{\partial t}=\alpha_{2} a-\mu_{\mathrm{p}} p,
$$

where $a$ is the level of ATP efficacy (dimensionless), $p$ is the concentration of $\mathrm{IP}_{3}$ $(\mu \mathrm{M}), \alpha_{1}$ is the rate constant of ATP efficacy reduction $\left(\mathrm{s}^{-1}\right), \alpha_{2}$ is the rate constant

\footnotetext{
${ }^{3}$ Throughout this paper we will use the notation of partial derivatives even though in some cases the derivatives are of functions which are only dependent on one variable and so the derivatives should be full derivatives. We have chosen to do this in order to make the extension of equations to include spatial dependence easier to read.
} 
of $\mathrm{IP}_{3}$ production per unit of ATP efficacy $\left(\mu \mathrm{Ms}^{-1}\right)$, and $\mu_{\mathrm{p}}$ is the rate constant of $\mathrm{IP}_{3}$ decay $\left(\mathrm{s}^{-1}\right)$, i.e. there is self-regulating decrease in overall $\mathrm{IP}_{3}$ production and this is taken into account using the constant $\mu_{\mathrm{p}}$.

We take as initial conditions: $a(0)=a_{0}$ and $p(0)=0$, where $a_{0}=3$ (dimensionless) is the initial efficacy of ATP proportional to the applied concentration of ATP (3 $\mu \mathrm{M})$.

Whilst throughout this paper it would be simple to use a numerical fitting procedure to obtain values for parameters from the data presented in Hirose et al. (1999) this would give a false sense of security. The data presented in Hirose et al. (1999) is representative data from a small number of experiments. This suggests there were quantitative variations from experiment to experiment, although no quantitative measure of the variations is given. Therefore, throughout this paper we will use values of parameters that give a good qualitative fit to the data in Hirose et al. (1999). By comparing the solutions for the equations above (subject to initial conditions) to the experiments, we find that the values $\alpha_{1}=0.05 \mathrm{~s}^{-1}, \alpha_{2}=0.05 \mu \mathrm{M} \mathrm{s}^{-1}$ and $\mu_{\mathrm{p}}=0.009 \mathrm{~s}^{-1}$ (see our Fig. 2A solid line), give good qualitative agreement between model and experiments. Although there have not been independent estimations of the parameters above, the value of the parameter $\mu_{\mathrm{p}}$ we are using is in the range that was used in previous theoretical studies (Meyer and Stryer, 1988, 1991; Atri et al., 1993).

We model the increase in cytosolic concentration of calcium upon application of extracellular calcium using the cell membrane permeability parameter $\beta$, which we find by fitting the data of Hirose et al. (1999). Thus, we take

$$
\frac{\partial c}{\partial t}=\beta\left(c_{\mathrm{e}}-c\right) \approx \beta c_{\mathrm{e}}=\mathrm{constant} \text { for } c \lll c_{\mathrm{e}},
$$

with $c(T)=0$ and $c_{\mathrm{e}}=100 \mu \mathrm{M}$, where $c$ is the concentration of cytosolic calcium, $c_{\mathrm{e}}$ is the extracellular concentration of calcium, and $T$ the time of extracellular calcium application. We are assuming here that the extracellular calcium in the culture medium is very high and the amount flowing into the cell is small so that we can consider the extracellular concentration of calcium constant in time. Taking the value for the parameter $\beta$ to be $5 \times 10^{-5} \mathrm{~s}^{-1}$ gave a good qualitative and quantitative fit to the data (see blue line on Fig. $2 \mathrm{~B}$ and dotted-dashed line on Fig. 2A).

The next step in developing the model is to modify the equation for $p$ in (1) to include the effect of calcium on $\mathrm{IP}_{3}$ levels. As mentioned earlier, there are several models that include feedback of calcium on $\mathrm{IP}_{3}$ production (Meyer and Stryer, 1988, 1991; Höfer et al., 2002). The feedback in those models is always positive. For example, the feedback term of Höfer et al. (2002) is $k_{1} c^{2} /\left(k_{2}^{2}+c^{2}\right)$ ( $k_{1}$ and $k_{2}$ are positive constants) and the feedback in Meyer and Stryer (1988, $1991)$ is $k_{3} c /\left(k_{4}+c\right)\left(k_{3}\right.$ and $k_{4}$ are positive constants). ${ }^{4}$ However, the data of Hirose et al. (1999) shows that monotonically increasing calcium concentration can give much more complex feedback on $\mathrm{IP}_{3}$ concentration. In particular, at low

\footnotetext{
${ }^{4}$ See also Fig. 3 for simulations conducted with these feedback terms and parameters presented in these papers.
} 
calcium concentration the feedback is mainly positive, and at high concentrations the feedback is negative. Thus, we will consider the additive form of feedback given by $f(c, p)=g_{1}(c)-g_{2}(c) p$, where we require that $g_{1}>g_{2}$ for small $c$ and $g_{2}>g_{1}$ for large $c$. The simplest possible form of $g_{1}$ and $g_{2}$ are $k_{p 1} c^{n}$ and $k_{p 2} c^{n+1}$, respectively. In addition to the previous conditions we will require that at the calcium steady state $c \leq 0.1 \mu \mathrm{M}$, the steady-state value of $\mathrm{IP}_{3}$ is also in a biologically realistic range, i.e. $p^{*} \leq 1 \mu \mathrm{M}$. Thus, we take the new $\mathrm{IP}_{3}$ equation to be

$$
\frac{\partial p}{\partial t}=\alpha_{2} a+k_{p 1} c^{n}-k_{p 2} c^{n+1} p-\mu_{\mathrm{p}} p
$$

We found that the values $n=2, k_{p 1}=1.125\left(\mu \mathrm{M}^{-1} \mathrm{~s}^{-1}\right)$ and $k_{p 2}=1.5\left(\mu \mathrm{M}^{-3} \mathrm{~s}^{-1}\right)$ gave sufficiently similar behaviour to that observed in the experiments of Hirose et al. (1999).

We believe that the model and parametrisation presented above adequately mimic the dynamics of $\mathrm{IP}_{3}$ and its up- and downregulation by changes in the levels of cytosolic calcium and ATP (see Fig. 2). As the calculations shown in Fig. 2 were done for the case when the internal calcium stores were abolished by ionomycin treatment, the observed calcium dynamics upon the application of extracellular calcium was very simple. However, when there are internal stores of calcium present on the ER, one would expect that the interplay between the calcium and $\mathrm{IP}_{3}$ dynamics will give rise to more complex dynamics, such as simultaneous oscillations of both $\mathrm{Ca}^{2+}$ and $\mathrm{IP}_{3}$, as shown in Fig. 3A of Hirose et al. (1999) (see reproduction in Fig. 4B).

\section{Simultaneous oscillations in $\mathrm{Ca}^{2+}$ and $\mathrm{IP}_{3}$ upon ATP stimulation}

As discussed in the previous section, we believe we have found a sufficient minimal model that mimics the dynamics of $\mathrm{IP}_{3}$ in response to stimulation by ATP and in response to increase in cytosolic calcium concentration. However, the increase in cytosolic calcium alone is not sufficient to produce the oscillations observed in Fig. 3A of Hirose et al. (1999) (reproduced in Fig. 4B in this paper). Calcium oscillations are thought to be caused by the periodic pumping of calcium ions between the cytoplasm and endoplasmic reticulum (ER), i.e. $\mathrm{Ca}^{2+}$ and $\mathrm{IP}_{3}$ can both activate $\mathrm{IP}_{3}$ receptors that release calcium from the ER, and at the same time the calcium in the cytoplasm can be pumped back to the ER. The existing models that predict simultaneous oscillations of $\mathrm{Ca}^{2+}$ and $\mathrm{IP}_{3}$ are those of Meyer and Stryer, (1988), Meyer and Stryer, (1991) and Mishra and Bhalla (2002). The first two of these, Meyer and Stryer $(1988,1991)$, are what one can call minimal models, since they consider the interaction between $\mathrm{Ca}^{2+}$ and $\mathrm{IP}_{3}$ only. The paper by Mishra and Bhalla (2002) presents a model for complex dynamics of $\mathrm{IP}_{3}$ and describes a detailed biochemical reaction of the inositol phosphate metabolism pathway consisting of 135-138 different molecules in the signalling cascade. Thus, this model is only amenable to numerical simulation and no mathematical analysis can be presented (the paper does not present the full mathematical formulation of the model). Also, whilst Mishra and Bhalla (2002) do show the possibility that $\mathrm{IP}_{3}$ can 
oscillate, the $\mathrm{IP}_{3}$ oscillation was always around dominant slow time dynamics and therefore it differed significantly from the data in Hirose et al. (1999). Clearly, whilst a model such as that of Mishra and Bhalla (2002) that has over 100 state variables and even more input parameters could in some parameter regime exhibit the behaviour observed in the experiments of Hirose et al. (1999), this parameter regime has not been found. Indeed, finding the right parameter range may be computationally difficult given the multidimensional parameter space of more than 100 different parameters.

In contrast to these large-scale computational studies, the models by Atri et al. (1993); Sneyd et al. (1998b); Wilkins and Sneyd (1998) consider simpler models which assume a calcium-induced calcium release mechanism where calcium can activate the $\mathrm{IP}_{3}$ receptors on the ER to induce more calcium release from the ER. In these models, the level of $\mathrm{IP}_{3}$ itself is considered to be an input parameter. The authors are able to assume a constant level of $\mathrm{IP}_{3}$ in these models since the specific experimental setup they consider has identified that this is a reasonable assumption. Thus, in these models, depending on the value of $\mathrm{IP}_{3}$, the calcium will undergo oscillations which are not accompanied by oscillations in $\mathrm{IP}_{3}$. This type of behaviour is not observed in the data by Hirose et al. (1999), which show that calcium oscillations are accompanied by oscillations of $\mathrm{IP}_{3}$. On the other hand, in the models of Meyer and Stryer (1988, 1991) there are simultaneous oscillations of calcium and $\mathrm{IP}_{3}$, but the effect of calcium on $\mathrm{IP}_{3}$ was only to upregulate it. As we discussed in the previous section, based on the experiments of Hirose et al. (1999), high concentrations of calcium seem to have an inhibitory effect on $\mathrm{IP}_{3}$. Since the handling of calcium concentration in the models of Meyer and Stryer $(1988,1991)$ is similar to the models of Atri et al. (1993); Sneyd et al. (1998b); Wilkins and Sneyd (1998), we use the latter as a template and include the more complex interplay between $\mathrm{Ca}^{2+}$ and $\mathrm{IP}_{3}$ as described in the previous section. In Fig. 3A of Hirose et al. (1999) the oscillations in $\mathrm{Ca}^{2+}$ and $\mathrm{IP}_{3}$ are seen only in response to stimulation by ATP without any external calcium application. Thus, in this section we assume that the elevated level of calcium inside the cell during oscillation does not result in the leakage of calcium out into the extracellular space, i.e. we are assuming that the cell membrane permeability for calcium leaving the cell is small enough to be negligible.

Based on the discussion above we build a four component model considering the concentration of cytosolic calcium, $c$, the fraction of non-activated $\mathrm{IP}_{3}$ receptors, $h$, concentration of $\mathrm{IP}_{3}, p$, and the concentration of effective stimulatory ATP, $a$. Thus, after Wilkins and Sneyd (1998) and the previous section of this paper, the model becomes

$$
\begin{aligned}
\frac{\partial c}{\partial t} & =J_{\text {flux }}-J_{\text {pump }}, \\
\tau \frac{\partial h}{\partial t} & =\frac{k_{2}^{2}}{k_{2}^{2}+c^{2}}-h, \\
\frac{\partial p}{\partial t} & =\alpha_{2} a-\mu_{\mathrm{p}} p+k_{p 1} c^{2}-k_{p 2} c^{3} p,
\end{aligned}
$$


and

$$
\frac{\partial a}{\partial t}=-\alpha_{1} a .
$$

The flux of calcium from the ER to the cytoplasm through the $\mathrm{IP}_{3}$ receptors, $J_{\text {flux }}$, is given after Wilkins and Sneyd (1998) by

$$
J_{\text {flux }}=k_{\mathrm{F}} \mu(p)\left(b+\frac{(1-b) c}{k_{1}+c}\right) h,
$$

where

$$
\mu(p)=\mu_{0}+\left(1-\mu_{0}\right) p /\left(k_{\mu}+p\right),
$$

is the fraction of $\mathrm{IP}_{3}$-activated $\mathrm{IP}_{3}$ receptors. The term

$$
\left(b+\frac{(1-b) c}{k_{1}+c}\right) \text { for } 0 \leq b \leq 1,
$$

shows the activation of $\mathrm{IP}_{3}$ receptors by calcium, i.e. when $b=1$ calcium cannot activate $\mathrm{IP}_{3}$ receptors, and thus there is no calcium-induced calcium release.

The amount of calcium pumped from the cytoplasm back to the ER is given by

$$
J_{\text {pump }}=\frac{\gamma c^{n}}{k_{\gamma}^{n}+c^{n}},
$$

where $n$ is the Hill coefficient for the calcium pump. Different authors take different values for this Hill coefficient. For example Meyer and Stryer $(1988,1991)$, Sneyd et al. (1995, 1998b) use Hill coefficient $n=2$, but Atri et al. (1993); Sneyd et al. (1998a); Wilkins and Sneyd (1998); Kummer et al. (2000); Mishra and Bhalla (2002) use the Hill coefficient $n=1$. We have chosen to use $n=1$ throughout this paper. The model could clearly be adapted to the $n=2$ case, however the review of results of the other models which have been simulated suggests that the qualitative results will not differ significantly. Also, in the above model the $\mathrm{IP}_{3}$ receptor dynamics is assumed to occur on a slower timescale than calcium dynamics, i.e. the timescale for $\mathrm{IP}_{3}$ receptor dynamics $(h)$ is proportional to $\tau$ in Eq. (5).

We want to investigate the behaviour of Eqs. (4)-(7). We begin by finding the steady states $\left(c^{*}, h^{*}, p^{*}, a^{*}\right)$. Initially we will consider the steady states of $c$ and $h$ in the absence of variations in $p$, i.e. we take $\mu$ to be constant. The steady states of $c^{*}$ and $h^{*}$ in this situation are the intersection points of the null clines given by

$$
\begin{aligned}
& h=\frac{\gamma c^{2} /\left(k_{\gamma}^{2}+c^{2}\right)}{k_{\mathrm{F}} \mu\left(b+\frac{(1-b) c}{k_{1}+c}\right)}=f(c), \\
& h=\frac{k_{2}^{2}}{k_{2}^{2}+c^{2}}=g(c) .
\end{aligned}
$$


The two graphs, $g$ and $f$, can in general have four intersection points and thus there can be four steady states $\left(h^{*}, c^{*}\right)$ for a given value of $\mu$, of which only three steady states are biologically relevant. However, as Atri et al. (1993) found, there is for most parameter values only one positive real steady state present. Wilkins and Sneyd (1998) later obtained a similar result. However, there is a small region in parameter space wherein three positive steady states are present. This region can be detected in Fig. 1 of Wilkins and Sneyd (1998). Therefore, in this paper we will derive more rigorous conditions for the existence of three positive real steady states. It is important to know of the existence of this region since travelling wave fronts separating regions of low calcium concentration from regions of high calcium concentration may only exist in such regions. Such wavefronts are relevant to calcium wave propagation in muscle (Murray, 1993), but we do not expect that for biologically realistic parameter regimes we would have stable steady states of high calcium concentration in MDCK epithelial cells.

There will be exactly two steady states present when

$$
f(c)=g(c) \quad \text { and } \quad \frac{\mathrm{d} f}{\mathrm{~d} c}=\frac{\mathrm{d} g}{\mathrm{~d} c},
$$

and there are only two values of $\mu, \mu_{1}$ and $\mu_{2}$, for which the equations in (14) are satisfied. When $\mu_{1}<\mu<\mu_{2}$ there are three positive steady states present (see Fig. 5 for illustration). For the parametrisation presented in Wilkins and Sneyd (1998), the critical values of $\mu$ are $\mu_{1}=0.288$ and $\mu_{2}=0.289$ (see Fig. 5).

In the presence of calcium feedback on $\mathrm{IP}_{3}$ levels the analogous bifurcation diagram can be drawn with respect to the parameter $k_{\mathrm{F}}$, which represents the calcium flux when all $\mathrm{IP}_{3}$ receptors are active. As one can see, there is a wide region in the parameter space where there are three steady states present. The smallest steady state is always a linearly stable node, the middle steady state is always a linearly unstable node or spiral, and the highest steady state can be either a linearly unstable or stable spiral depending on the value of $k_{\mathrm{F}}$. Thus, the highest steady state undergoes a Hopf bifurcation as $k_{\mathrm{F}}$ decreases, leading to a limit cycle. As $k_{\mathrm{F}}$ decreases further the limit cycle collides with the unstable saddle to form a homoclinic orbit and then disappears. We show the maximum of the limit cycle by grey circles and minimum by black circles on Fig. 6. Whilst the standard model by Wilkins and Sneyd (1998) shows limit cycle oscillations for a wide range of values of the calcium release parameter, our model predicts that due to the calcium feedback to $\mathrm{IP}_{3}$ production, the limit cycles occur in a smaller range of parameter values.

Introducing $3 \mu \mathrm{M}$ of ATP at time zero generates the simultaneous oscillations shown in Fig. 4. As we can see in Fig. 4 the model is able to produce oscillations with similar periodicity to those presented in experiments (Fig. 4B), i.e. 7-8 peaks in $250 \mathrm{~s}$.

\section{Intracellular spatio-temporal dynamics of calcium and $\mathrm{IP}_{3}$}

Figure 3 (left) of Hirose et al. (1999) (reproduced in Fig. 7B) shows the intracellular dynamics of $\mathrm{IP}_{3}$ and $\mathrm{Ca}^{2+}$ in response to stimulation of the cell with $3 \mu \mathrm{M}$ of 
ATP. The stimulatory effect of ATP appears to be localised to a very small region at the ends of what looks like a very narrow and long cell. The cell in question is about $100 \mu \mathrm{m}$ long and $10 \mu \mathrm{m}$ wide. It appears that within $9 \mathrm{~s}$ of initiation the $\mathrm{Ca}^{2+}$ and $\mathrm{IP}_{3}$ levels are observed to go up and what appears to be uniform levels of $\mathrm{Ca}^{2+}$ and $\mathrm{IP}_{3}$ are obtained. We model this situation by extending the model presented in the previous section to include the spatial dynamics of $\mathrm{Ca}^{2+}$ and $\mathrm{IP}_{3}$, and include the stimulatory effect of ATP at the surface of the cell. Thus, inside the cell domain $\Omega$ we take

$$
\begin{aligned}
\frac{\partial c}{\partial t} & =D_{\mathrm{c}} \nabla^{2} c+J_{\text {flux }}-J_{\text {pump }}, \\
\tau \frac{\partial h}{\partial t} & =\frac{k_{2}^{2}}{k_{2}^{2}+c^{2}}-h, \\
\frac{\partial p}{\partial t} & =D_{\mathrm{p}} \nabla^{2} p-\mu_{\mathrm{p}} p+k_{p 1} c^{2}-k_{p 2} c^{3} p,
\end{aligned}
$$

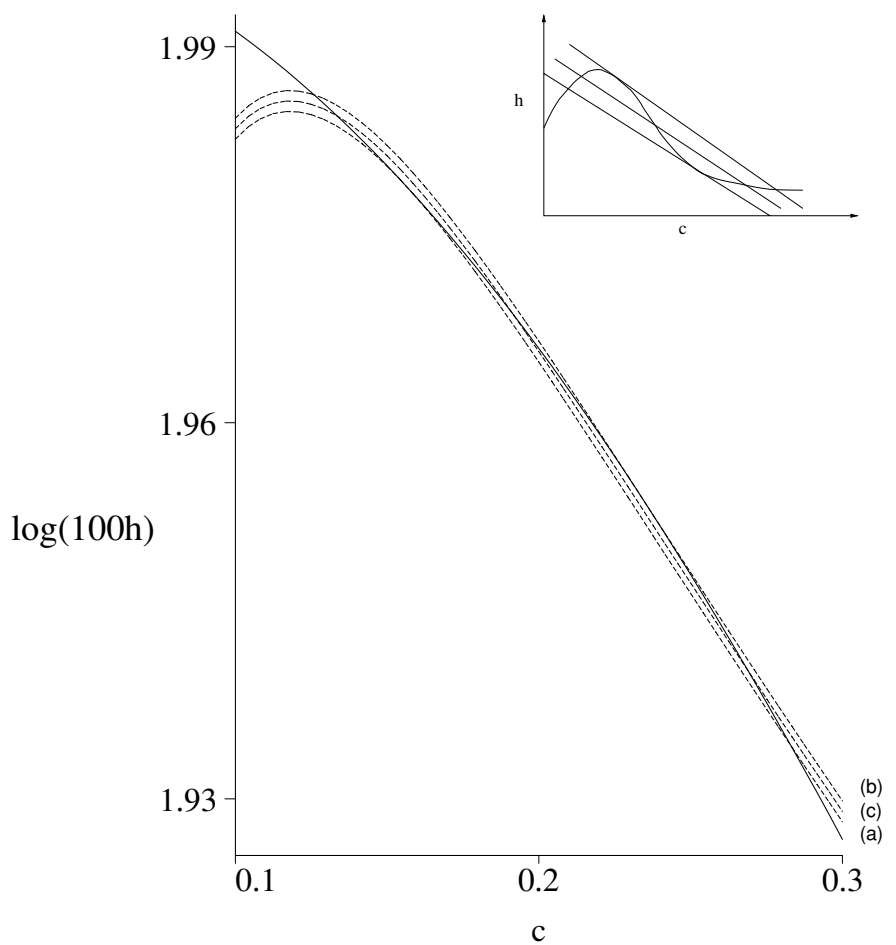

Fig. 5 Main graph: Null clines $f(c)$ (dotted lines) given by Eq. (12) and $g(c)$ (solid line) given by Eq. (13) for (a) $\mu=\mu_{1}$, (b) $\mu=\mu_{2}$ and (c) $\mu=\left(\mu_{1}+\mu_{2}\right) / 2$ using parameters presented in Wilkins and Sneyd (1998). Inset: Cartoon illustration for existence of three positive steady states. $h$ is defined as in equations (12) and (13). 

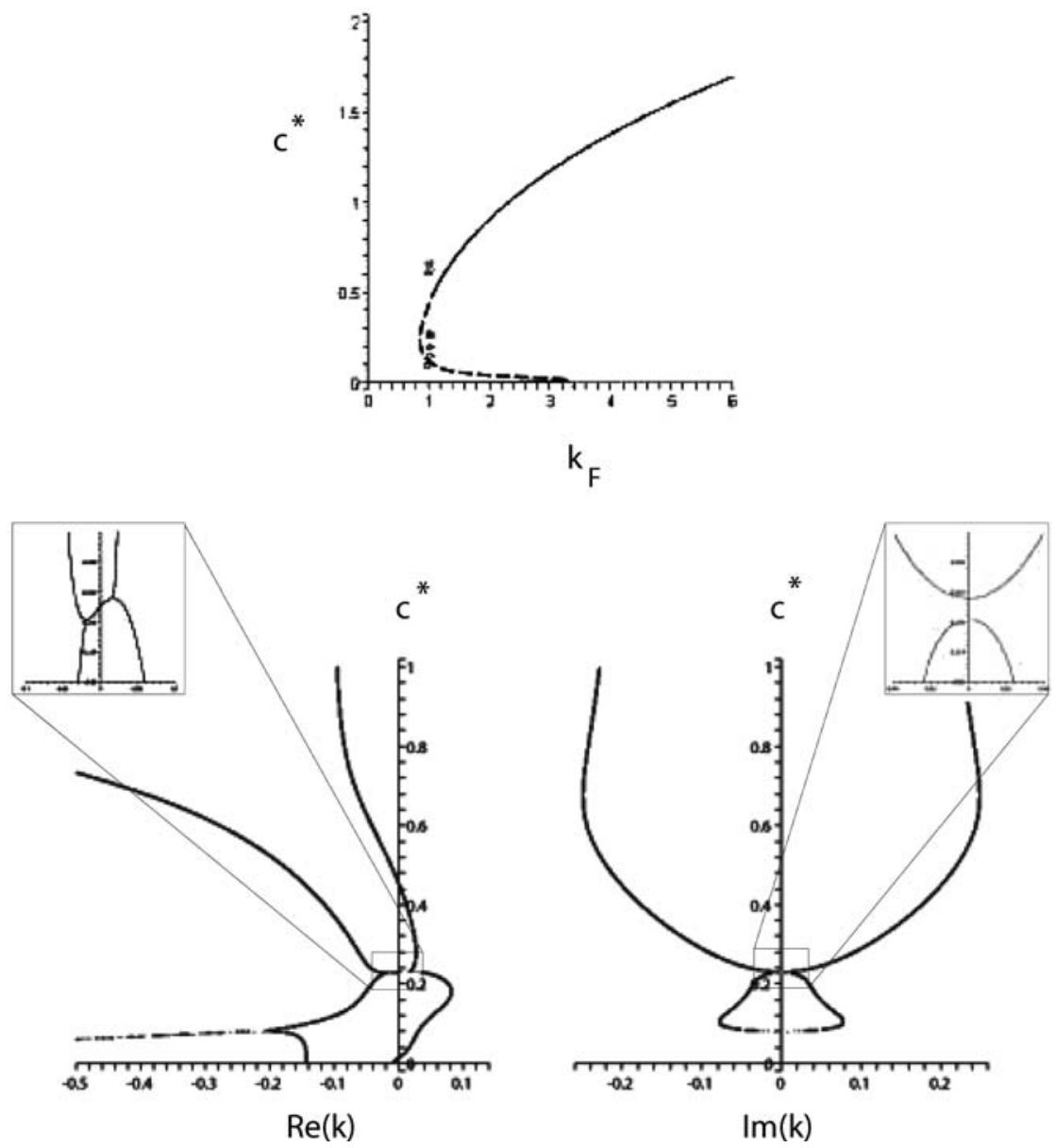

Fig. 6 Bifurcation graph for calcium steady state $c^{*}$ as function of $k_{\mathrm{F}}$ (top graph), the calcium flux parameter when all $\mathrm{IP}_{3}$ receptors are activated. Solid lines show linearly stable steady-state values and dashed lines linearly unstable steady states. Grey and black circles show maximum and minimum values of $c$ during the limit cycle, respectively. The bottom two graphs show real and imaginary parts of the eigenvalues $k$ as a function of the steady-state values $c^{*}$ corresponding to the top bifurcation graph with $k_{\mathrm{F}}$ changing.

where $D_{\mathrm{c}}$ is the diffusion coefficient for intracellular calcium, and $D_{\mathrm{p}}$ is the diffusion coefficient for intracellular $\mathrm{IP}_{3}$ (see Table 1 for parameter values). The stimulatory effect of ATP application on the boundary will be modelled as follows,

$$
-\left(D_{\mathrm{p}} \nabla p\right) \cdot \mathbf{n}=\lambda a(t) \quad \text { on } \quad \mathbf{r}=\partial \Omega,
$$

where $\partial \Omega$ is the cell boundary with normal $\mathbf{n}, \lambda$ is the amount (mol) of $\mathrm{IP}_{3}$ created per unit of cell surface area per unit time per unit of the measure of ATP efficacy, and $a(t)$ is the measure of ATP efficacy. We are assuming that the efficacy of ATP 

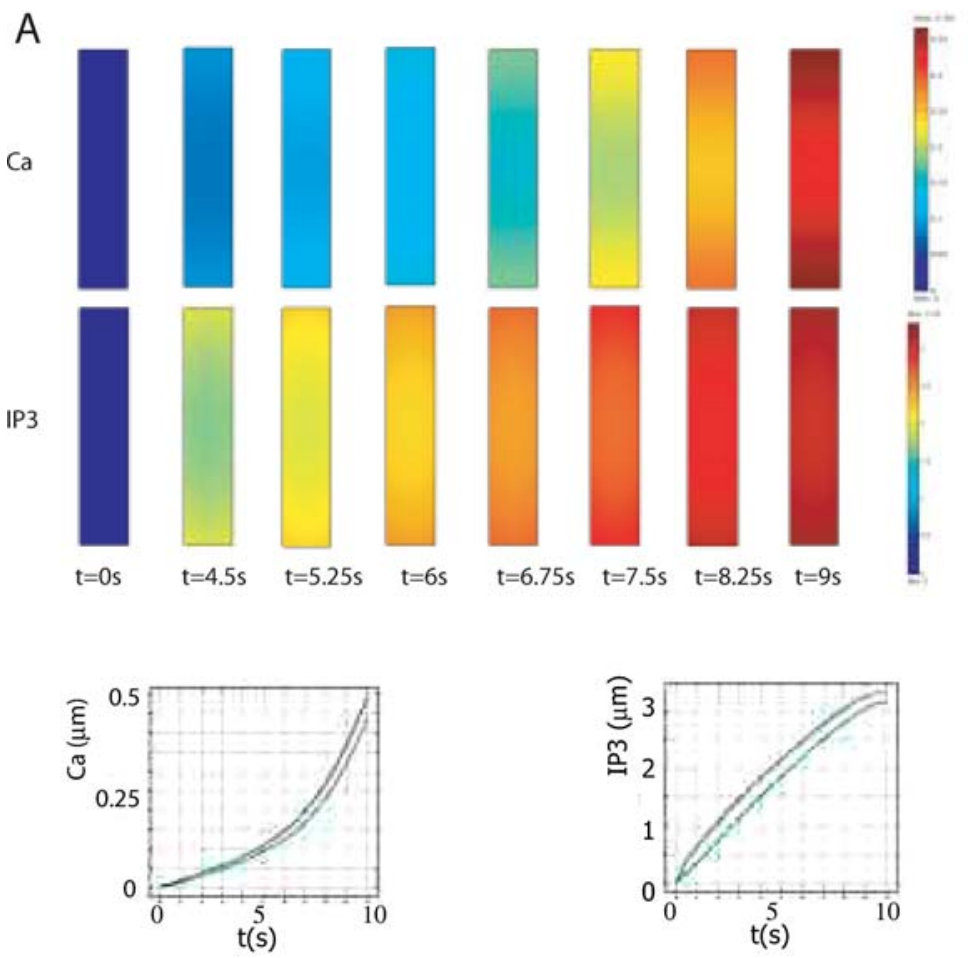

B

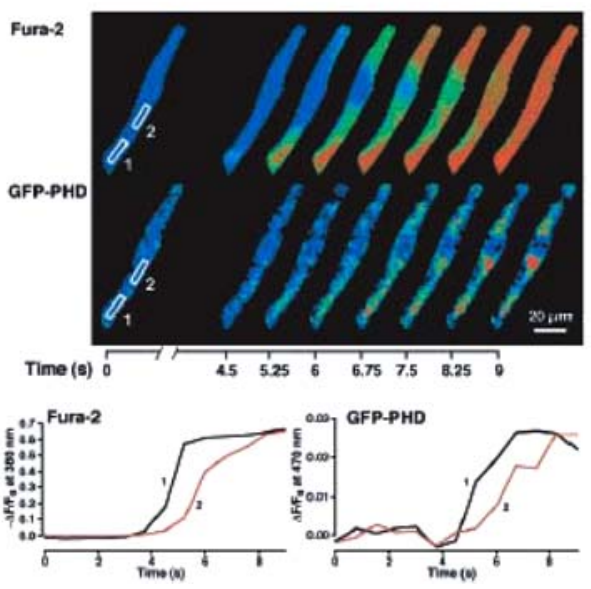

Fig. 7 (A) Intracellular $\mathrm{Ca}^{2+}$ and $\mathrm{IP}_{3}$ dynamics in a $10 \mu \mathrm{m} \times 100 \mu \mathrm{m}$ cell at times $t=$ 4.5, 5.25, 6, 6.75, 7.5, 8.25 and $9 \mathrm{~s}$ as predicted by the model given by Eqs. (15)-(17) solved using Femlab 3.1. The time-dependent traces are shown below and are taken from the tip (blue lines) and from the centre (green lines) of the cell. Numerical results in (A) correspond to experiments shown in (B) reproduced from Fig. 4 (left) of Hirose et al. (1999) (Reprinted with permission from Hirose et al. (1999) (Copyright 1999, AAAS).) 
Table 1 Model parameters.

\begin{tabular}{|c|c|c|c|c|}
\hline Parameter & Value & Unit & Reference & Definition \\
\hline$\alpha_{1}$ & 0.05 & $\mathrm{~s}^{-1}$ & (a) & Rate constant of ATP decay \\
\hline$\alpha_{2}$ & 0.05 & $\mu \mathrm{Ms}^{-1}$ & (a) & Rate of $\mathrm{IP}_{3}$ production by ATP \\
\hline$\mu_{\mathrm{p}}$ & 0.009 & $\mathrm{~s}^{-1}$ & (a) & Rate constant of $\mathrm{IP}_{3}$ degradation \\
\hline$T$ & 120 & $\mathrm{~s}$ & (a) & Time of extracellular $\mathrm{Ca}^{2+}$ application \\
\hline$\beta$ & $5 \times 10^{-3}$ & $\mathrm{~s}^{-1}$ & (a) & $\begin{array}{l}\text { Rate constant of extracellular } \\
\text { calcium internalisation }\end{array}$ \\
\hline$k_{p 1}$ & 1.125 & $\mu \mathrm{M}^{-1} \mathrm{~s}^{-1}$ & (a) & $\begin{array}{l}\text { Rate constant of } \mathrm{Ca}^{2+} \text { positive } \\
\text { feedback on } \mathrm{IP}_{3}\end{array}$ \\
\hline$k_{p 2}$ & 1.5 & $\mu \mathrm{M}^{-3} \mathrm{~s}^{-1}$ & (a) & $\begin{array}{l}\text { Rate constant of } \mathrm{Ca}^{2+} \text { negative } \\
\text { feedback on } \mathrm{IP}_{3}\end{array}$ \\
\hline$k_{\mathrm{F}}$ & 1.034 & $\mu \mathrm{Ms}^{-1}$ & (a) & $\begin{array}{l}\mathrm{Ca}^{2+} \text { flux when all } \mathrm{IP}_{3} \\
\text { receptors are open }\end{array}$ \\
\hline$b$ & 0.111 & - & (b) & $\begin{array}{l}\text { Fraction of open } \mathrm{IP}_{3} \\
\quad \text { receptors when }\left[\mathrm{Ca}^{2+}\right]=0\end{array}$ \\
\hline$\gamma$ & 0.286 & $\mu \mathrm{Ms}^{-1}$ & (b) & $\begin{array}{l}\text { Max. rate of } \\
\mathrm{Ca}^{2+} \text { pumping to ER }\end{array}$ \\
\hline$k_{\gamma}$ & 0.1 & $\mu \mathrm{M}$ & (b) & $\begin{array}{l}\text { Michaelis-Menten coefficient for } \\
\mathrm{Ca}^{2+} \text { pump to ER }\end{array}$ \\
\hline$k_{2}$ & 0.7 & $\mu \mathrm{M}$ & (b) & $\begin{array}{l}\text { Michaelis-Menten coefficient for } \\
\mathrm{IP}_{3} \mathrm{R} \text { activation }\end{array}$ \\
\hline$\tau$ & 7 & $\mathrm{~s}$ & (a) & $\begin{array}{l}\mathrm{IP}_{3} \text { receptor } \\
\text { inactivation timeconstant }\end{array}$ \\
\hline$k_{1}$ & 0.7 & $\mu \mathrm{M}$ & (b) & $\begin{array}{l}\text { Michaelis-Menten coefficient for } \\
\mathrm{Ca}^{2+} \text {-induced } \mathrm{Ca}^{2+} \text { release }\end{array}$ \\
\hline$k_{\mu}$ & 0.7 & $\mu \mathrm{M}$ & (a) & $\begin{array}{l}\text { Michaelis-Menten coefficient } \\
\text { for } \mathrm{IP}_{3} \text { in } \mu\end{array}$ \\
\hline$\lambda$ & 1.25 & $\mu \mathrm{m} \mathrm{s}^{-1}$ & (a) & $\begin{array}{l}\text { Rate constant of } \mathrm{IP}_{3} \text { production by } \\
\text { ATP per unit cell surface area }\end{array}$ \\
\hline$\mu_{0}$ & 0.05 & & (a) & $\begin{array}{l}\text { Proportion of activated } \mathrm{IP}_{3} \mathrm{R} \\
\quad \text { when }\left[\mathrm{IP}_{3}\right]=0\end{array}$ \\
\hline$D_{\mathrm{c}}$ & 20 & $\mu \mathrm{m}^{2} \mathrm{~s}^{-1}$ & (c) & $\begin{array}{l}\text { Rate of intracellular } \\
\mathrm{Ca}^{2+} \text { diffusion }\end{array}$ \\
\hline$D_{\mathrm{p}}$ & 300 & $\mu \mathrm{m}^{2} \mathrm{~s}^{-1}$ & (c) & $\begin{array}{l}\text { Rate of intracellular } \\
\mathrm{IP}_{3} \text { diffusion }\end{array}$ \\
\hline$M_{0}$ & 0.1 & $\mu \mathrm{Ms}^{-1}$ & (a) & $\begin{array}{l}\text { Rate of } \mathrm{Ca}^{2+} \text { release due to } \\
\text { mechanical stimulation }\end{array}$ \\
\hline$N_{0}$ & 3.75 & $\mu \mathrm{M} \mathrm{s}^{-1}$ & (a) & $\begin{array}{l}\text { Rate of } \mathrm{IP}_{3} \text { release due to } \\
\text { mechanical stimulation }\end{array}$ \\
\hline$t_{\text {crit }}$ & 7.5 & $\mathrm{~s}$ & (a) & Time of mechanical action \\
\hline
\end{tabular}

Note. References: (a) Our estimation based on data in Hirose et al. (1999), (b) Atri et al. (1993), (c) Allbritton et al. (1992).

is not spatially dependent, but it is 'well mixed' and that the efficacy of ATP decays with a rate constant $\alpha_{1}$ defined earlier, i.e. $\partial a / \partial t=-\alpha_{1} a$, and thus $a(t)=a(0) e^{-\alpha_{1} t}$. Thus, we are assuming that it is not the ATP efficacy gradient or the ATP concentration gradient that is driving the intracellular calcium and $\mathrm{IP}_{3}$ dynamics.

The boundary condition for $\mathrm{Ca}^{2+}$ concentration $c$ is given by

$$
-\left(D_{\mathrm{c}} \nabla c\right) \cdot \mathbf{n}=0 \quad \text { on } \quad \mathbf{r}=\partial \Omega
$$




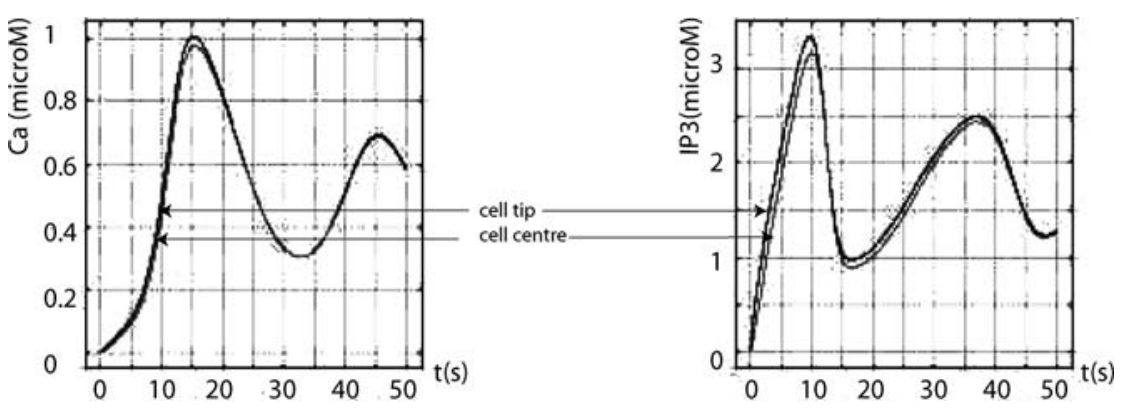

Fig. 8 Long-time behaviour of intracellular $\mathrm{Ca}^{2+}$ and $\mathrm{IP}_{3}$ in a long cell shown in Fig. 7. We see that after initial transients, the model predicts that the cell will enter an oscillatory regime.

i.e. no flux of calcium through the cell membrane. This boundary condition assumes that the transfer of calcium between the inside of the cell and the outside is slow. As there is no excess extracellular application of calcium we do not envisage flux of calcium into the cell. However, following the stimulation of the cells with ATP and the resulting elevations in $\mathrm{IP}_{3}$ and $\mathrm{Ca}^{2+}$ inside the cell there might be a flux of $\mathrm{Ca}^{2+}$ from the cell to extracellular space. With the boundary condition given above we are assuming that this outward flux of calcium is small.

We choose the initial condition for $\mathrm{Ca}^{2+}, \mathrm{IP}_{3}$, and $\mathrm{IP}_{3} \mathrm{R}$ to be given by the steady-state value in the absence of stimulation. We choose the parameters in the model so that there is a single steady state that is linearly stable. This ensures that the introduction of diffusion does not give rise to travelling waves or oscillations in the absence of stimulation by ATP. In Hirose et al. (1999) the 'travelling waves' are seen only when there is external stimulation present and the background levels of calcium are assumed to be stable to arbitrary small fluctuations.

We can see in Fig. 7 that the difference in the levels of $\mathrm{Ca}^{2+}$ and $\mathrm{IP}_{3}$ agree reasonably well with the experimental observations. However, the profiles that develop are not what one would call classical travelling wave profiles. Unlike in the experiments, where the data is only recorded up to $10 \mathrm{~s}$ after stimulation, we can predict the behaviour of the cell at longer timescales using our model. As one can see in Fig. 8, after the initial increase of $\mathrm{Ca}^{2+}$ and $\mathrm{IP}_{3}$ the model predicts that the cell will enter into an oscillatory mode which will persist with the levels of $\mathrm{Ca}^{2+}$ and $\mathrm{IP}_{3}$ being essentially homogenous inside the cell for times larger than $50 \mathrm{~s}$ (see Fig. 8 for temporal values at the edge of the cell and in the middle of the cell). The long time temporal variation in $\mathrm{Ca}^{2+}$ and $\mathrm{IP}_{3}$ levels is indeed in good qualitative agreement with the data in Fig. 4. Using the model we can also investigate the difference between long cells and short cells; for example in Fig. 9 we present numerical results for a cell which is $10 \mu \mathrm{m} \times 10 \mu \mathrm{m}$. As expected, the edge of the cell and the centre of the cell reach the synchronous oscillatory phase much faster than in the case of the long cell. 

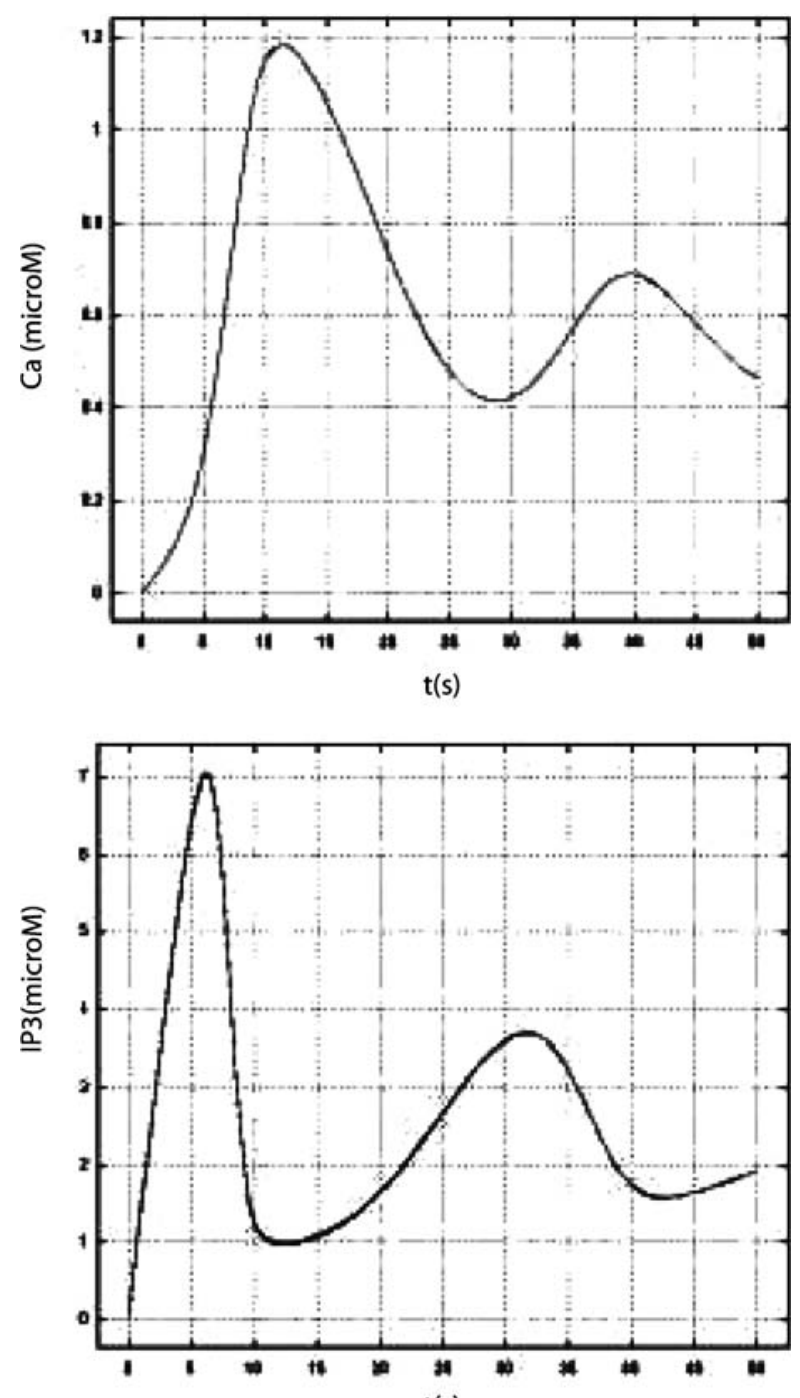

$t(s)$

Fig. 9 Intracellular $\mathrm{Ca}^{2+}$ and $\mathrm{IP}_{3}$ dynamics for a short $10 \mu \mathrm{m}$ long cell. Cell tip and cell centre rapidly reach the same levels, and thus the cell is oscillating uniformly without any intracellular spatial variation in $\mathrm{Ca}^{2+}$ and $\mathrm{IP}_{3}$ levels. The lines for cell tip and cell centre are overlapping since in the small cell there are no intracellular calcium and $\mathrm{IP}_{3}$ gradients. The cell tip and centre concentrations rapidly come into equilibrium, and thus the lines on the graphs overlap.

\section{Intercellular spatio-temporal dynamics of calcium and $\mathrm{IP}_{3}$}

Now that we have developed a model for describing the spatio-temporal dynamics of $\mathrm{Ca}^{2+}$ and $\mathrm{IP}_{3}$ inside a single cell, we turn our attention to the factors affecting the intercellular dynamics of these two chemical messengers. It is clear that this is 
the main point of concern in biology as multicellular organs can have quite complex intercellular patterns for calcium signalling. Intercellular calcium signalling is known to play a role in a wide variety of biological situations such as the functioning of heart, brain and muscles (Berridge et al., 1998, 2000; Marks, 2003), and also on the development of embryos (Wallingford et al., 2002), and in gene expression (Dolmetsch et al., 1998).

Hirose et al. (1999) conducted an experiment to observe the simultaneous intercellular $\mathrm{Ca}^{2+}$ and $\mathrm{IP}_{3}$ waves in a monolayer of cells. In this section we will present a model that builds on the models presented in previous sections of this paper for the experimental situation of Hirose et al. (1999). However, before proceeding, there are several points that we need to discuss concerning the data Hirose et al. (1999) present.

The cells in the confluent monolayer in the experiments of Hirose et al. (1999) appear to be much smaller than the cells in isolation. The approximate crosssection of the cell in isolation was about $10 \mu \mathrm{m} \times 100 \mu \mathrm{m}$, but the cells in the confluent monolayer appear to be $15 \mu \mathrm{m} \times 15 \mu \mathrm{m}$ in cross-section. It would be interesting to know if this is due to the cell packing which gives rise to thicker cells in a confluent monolayer as opposed to more spread out cells in isolation, i.e. is the single cell volume conserved between Fig. 4 (left) and (right) of Hirose et al. (1999)? Change in cell volume could of course have influence on cell internal biochemical reactions as the concentrations of chemical messengers inside smaller cells might be higher than inside larger cells. This would be consistent with several observations where mechanical manipulation of cells resulted in differences in gene regulation, movement in mRNA, etc. (Chicurel et al., 1998; Fargem, 2003). Therefore, it could be that intrinsic calcium and $\mathrm{IP}_{3}$ signalling might be different between cells in isolation and cells in tight monolayers. However, for simplicity we assume that cell monolayers are the same as isolated cells.

The second point that has not been answered in Hirose et al. (1999) concerns the causal relationship between the intercellular $\mathrm{Ca}^{2+}$ and $\mathrm{IP}_{3}$ waves and the mechanical stimulation used to initiate them. It is an open question whether in epithelial cells the mechanical stimulation releases $\mathrm{Ca}^{2+}$ from $\mathrm{IP}_{3}$-independent calcium stores or whether the mechanical stimulation induces $\mathrm{IP}_{3}$ production itself. Several authors have found in carefully controlled experiments that calcium levels are increased from an $\mathrm{IP}_{3}$ insensitive reservoir by stretch or shear of cells (Blackman et al., 2000; Mohanti et al., 2001; Li, 2002). Similarly, it has been found that the levels of $\mathrm{IP}_{3}$ can go up as a result of cell stretch (Felix et al., 1996). However, the problem of translating these carefully obtained results into the experimental setup described in Hirose et al. (1999) is not straightforward. Essentially, we do not know quantitatively what happens during mechanical stimulation; we do not know the size of the stimulatory area, duration of the stimulatory event, or the stress/strain that was applied to achieve the observed intercellular $\mathrm{Ca}^{2+}$ and $\mathrm{IP}_{3}$ waves. We also do not know what is the interplay between $\mathrm{Ca}^{2+}$ and $\mathrm{IP}_{3}$ during the mechanical stimulation of the cell, i.e. do they act cooperatively or are their responses to mechanical stimulation independent of each other? It is suggested (Hirose personal communication) that $\mathrm{Ca}^{2+}$ and $\mathrm{IP}_{3}$ act cooperatively in response to mechanical stimulation, i.e. no $\mathrm{IP}_{3}$ or $\mathrm{Ca}^{2+}$ waves were observed in cells that had internal calcium stores abolished. In this paper, we assume that the mechanical 
stimulation acts over the cross-sectional area of a single cell and we choose the duration and magnitude of $\mathrm{Ca}^{2+}$ and $\mathrm{IP}_{3}$ upregulation to match the observed experimental results.

In the previous models that deal with mechanically induced intercellular calcium waves, the mechanical representation has been modelled by introducing a constant source term of $\mathrm{IP}_{3}$ for a specified amount of time (Sneyd et al., 1995; Atri et al., 1993). We will take a similar approach, but also include the fact that calcium itself can be directly upregulated by the mechanical manipulation of the cell.

Finally, several researchers have found that the intercellular waves are slower than intracellular waves due to movement of chemical messengers across cell membranes. In particular, gap junctions are known to slow down the movement of $\mathrm{IP}_{3}$ from cell to cell (Boitano et al., 1992; Sneyd et al., 1995). In order to model gap junctions authors generally use internal boundaries and relate the flux across these boundaries to the permeability of the gap junction and difference in concentration on either side of the boundary (Sneyd et al., 1995; Höfer et al., 2002). Alternatively, if a wave is travelling over many cells, we can use homogenisation theory and find an effective diffusion coefficient across the tissue to be given by $D_{\text {eff }}=D K L /(D+K L)$, where $D$ is diffusion coefficient within a single cell, $K$ is the permeability of gap junctions, and $L$ is the distance between the gap junctions (Keener and Sneyd, 1998). In the experimental set-up of Hirose et al. (1999) shown in Fig. 10, the cell boundaries stand out as preferred locations of the wavefronts, which is usually the case where the waves 'pause' before propagating to the next cell, indicating that the gap junctions slow down the wave. On the other hand the overall speed on the intercellular wave in Fig. 10 is similar to that of the intracellular wave in Fig. 7. For simplicity we use the effective diffusion coefficient approach, and in the absence of any detailed experimental data on the permeability of gap junctions for MDCK epithelial cells in the experiments of Hirose et al. (1999) we take $D_{\text {eff }}=D$.

Thus, we take the model for intercellular $\mathrm{Ca}^{2+}$ and $\mathrm{IP}_{3}$ movement to be essentially the same as shown in the previous section for intracellular waves, but with small modifications to account for the mechanical stimulation, i.e. we take the equations to be

$$
\begin{aligned}
\frac{\partial c}{\partial t} & =D_{\mathrm{c}} \nabla^{2} c+J_{\text {flux }}-J_{\text {pump }}+M_{\text {stim }}, \\
\tau \frac{\partial h}{\partial t} & =\frac{k_{2}^{2}}{k_{2}^{2}+c^{2}}-h, \\
\frac{\partial p}{\partial t} & =D_{\mathrm{p}} \nabla^{2} p-\mu_{\mathrm{p}} p+k_{p 1} c^{2}-k_{p 2} c^{3} p+N_{\mathrm{stim}},
\end{aligned}
$$

where $M_{\text {stim }}$ and $N_{\text {stim }}$ are, respectively, the rate of $\mathrm{Ca}^{2+}$ and $\mathrm{IP}_{3}$ release due to mechanical stimulation. In the equations above we are assuming that the $\mathrm{Ca}^{2+}$ and $\mathrm{IP}_{3}$ release is independent of $\mathrm{IP}_{3}$ receptors and that this process is additive, i.e. that the mechanical stimulation introduces new source terms for $\mathrm{Ca}^{2+}$ and $\mathrm{IP}_{3}$, but does not influence the $\mathrm{Ca}^{2+}$ and $\mathrm{IP}_{3}$ release rate constants. If it was to be found that the $\mathrm{Ca}^{2+}$ is released from the $\mathrm{IP}_{3}$ receptor sensitive pool then the equations above 

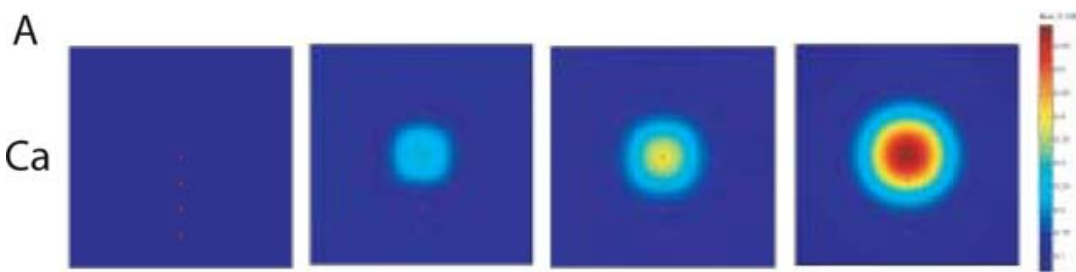

IP3
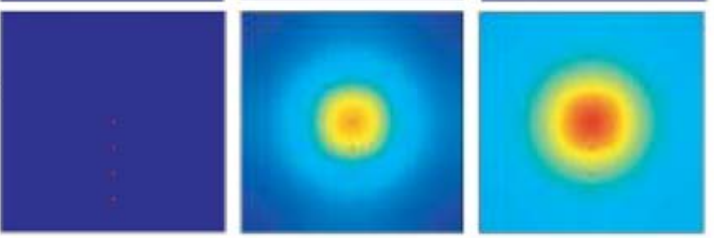

$\mathrm{t}=0 \mathrm{~s}$

$t=3 s$

$t=4.5 \mathrm{~s}$

$\mathrm{t}=7.5 \mathrm{~s}$

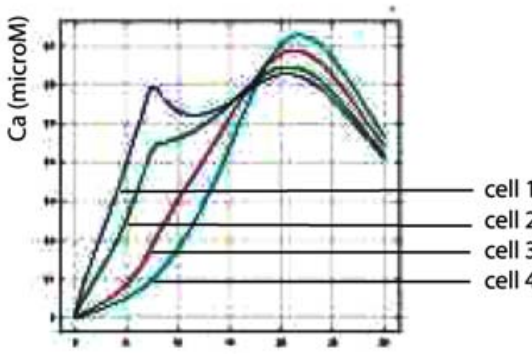

$t(s)$

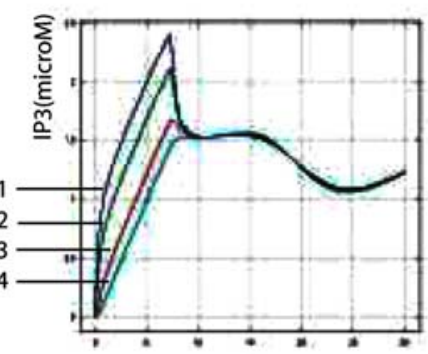

$\mathrm{t}(\mathrm{s})$

B
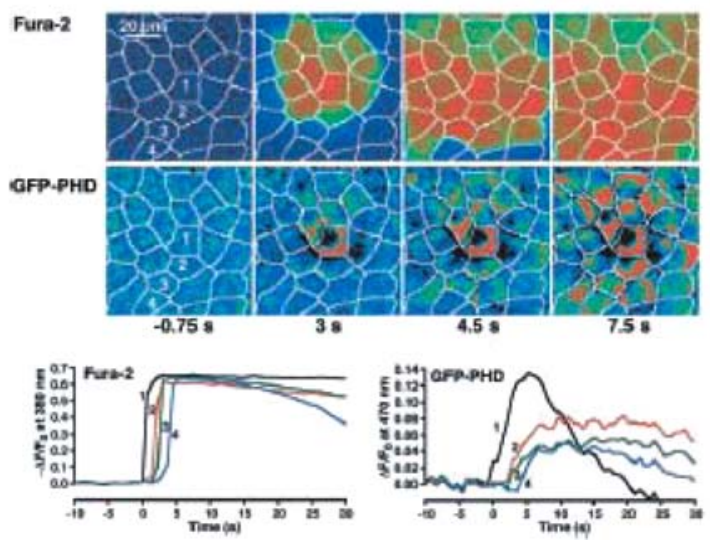

Fig. 10 (A) Intercellular $\mathrm{Ca}^{2+}$ and $\mathrm{IP}_{3}$ dynamics with $\mathrm{Ca}^{2+}$ and $\mathrm{IP}_{3}$ upregulation due to mechanical stimulation. Mechanical stimulation induces upregulation in $\mathrm{IP}_{3}$ and in $\mathrm{Ca}^{2+}$, i.e. $M_{0}=0$ and $N_{0}=2.5 \mu \mathrm{M} \mathrm{s}^{-1}$ in (23) and (24). The model, given by Eqs. (20)-(24), was solved using Femlab 3.1. (B) Experimental data reprinted with permission from Hirose et al. (1999) (Copyright 1999, AAAS). 
would need to be modified to take the mechanical stimulation-induced increase in $k_{\mathrm{F}}$ (the rate parameter in $J_{\text {flux }}$ ) into account. However, in this paper we stick with the first approach as there is no evidence that the behaviour of the $\mathrm{IP}_{3}$ receptors is modified during mechanical stimulation.

We take the forms of the terms $M_{\text {stim }}$ and $N_{\text {stim }}$ to be

$M_{\text {stim }}=M_{0}, \quad$ for $\quad 0<r<r_{\text {cell }}, 0<t<t_{\text {crit }} \quad$ and $\quad M_{\text {stim }}=0 \quad$ elsewhere,

and

$N_{\text {stim }}=N_{0}, \quad$ for $\quad 0<r<r_{\text {cell }}, 0<t<t_{\text {crit }}$ and $\quad N_{\text {stim }}=0$ elsewhere,

where $r_{\text {cell }}$ is the radius of the cell that is being stimulated, $t_{\text {crit }}$ is the duration of the stimulatory effects and $M_{0}$ and $N_{0}$ are the magnitudes of the stimulatory effects.

We have run an extensive parametrisation study to determine which values of $N_{0}, M_{0}$ and $t_{\text {crit }}$ would give the results that most resemble experimental data. In doing this type of parametrisation, when varying $t_{\text {crit }}$ we also always varied $N_{0}$ and $M_{0}$ so as to not allow the calcium and $\mathrm{IP}_{3}$ concentrations to go outside the range seen in the intracellular modelling results and experimental observations.

In Fig. 10, we show the results that most resemble the experimental observations of Hirose et al. (1999). As can be seen, our model results are in good agreement with the experimental observations at early times, i.e. for times less than $10 \mathrm{~s}$. Our model predicts that one should see the upregulation of $\mathrm{Ca}^{2+}$ and $\mathrm{IP}_{3}$ within $10 \mathrm{~s}$ of stimulation. This is consistent with the experiments presented in Hirose et al. (1999). However, after $20 \mathrm{~s}$ we should see the lowering of $\mathrm{IP}_{3}$ levels followed by somewhat slower recovery while $\mathrm{Ca}^{2+}$ should exhibit a more monotone behaviour. Also, in the experiments the level of $\mathrm{IP}_{3}$ in the mechanically stimulated cell drops below the prestimulated level without being accompanied by similar downregulation in calcium. There might be several reasons for the observed discrepancies between experiments and model. Firstly, as noted before, we do not know what happens during mechanical stimulation of the cell. We do not know the rate at which $\mathrm{Ca}^{2+}$ and $\mathrm{IP}_{3}$ release occurs, and indeed we do not know if another chemical messenger is involved in this process. Therefore, the way we have modelled mechanical stimulation might not be appropriate even though it is consistent with all of the previous models (Atri et al., 1993; Sneyd et al., 1995; Höfer et al., 2002). Secondly, the importance of cell packing and the large differences in apparent cell morphology between the cells in isolation and in confluent monolayer could play a role in this system. Finally, the experiments were done by recording the changes in the fluorescence intensity coming from the $\mathrm{Ca}^{2+}$ that was tagged with fluorophore. One might speculate that the fluorescence signal in these experiments was saturated since the calcium data observed in Fig. 4 (right) of Hirose et al. (1999) reaches a fast plateau level where the data is less noisy than in the other experiments. Clearly, no dynamics can be measured if the detector is saturated. 


\section{Discussion}

In this paper, we set out to develop a preliminary mathematical model that would capture the interplay between $\mathrm{Ca}^{2+}$ and $\mathrm{IP}_{3}$ signalling shown in the experimental paper by Hirose et al. (1999). We believe our model to be compatible with experiments on the intracellular scale. Using mathematical modelling we were able to pinpoint the problems that should be tackled experimentally before refining the model any further. In the absence of any detailed physiological information on the way in which calcium regulates $\mathrm{IP}_{3}$ we chose a simple functional form which matched the available experimental data. Thus, the model is preliminary and will need to be refined as more detailed biological information about the relationship between $\mathrm{IP}_{3}$ and calcium becomes available.

The main prediction of the model is that in addition to calcium upregulating $\mathrm{IP}_{3}$ at low calcium levels, it also has to actively downregulate the $\mathrm{IP}_{3}$ levels at high calcium levels. A simple reduction in the rate of $\mathrm{IP}_{3}$ upregulation with increasing calcium concentration is not enough as it will not ensure the stability of a physiologically realistic unstimulated steady state. This prediction directly motivates future experiments to look at the effect of the rapid increase in intracellular calcium on the levels of $\mathrm{IP}_{3}$. This could be done by releasing stored calcium within the cell to achieve much faster rises in intracellular calcium levels than were observed in Hirose et al. (1999). This experiment would give an indication as to whether it is the transient behaviour of calcium or the absolute value of the calcium concentration that provides the switching mechanism for $\mathrm{IP}_{3}$.

It remains an open question as to what happens during mechanical stimulation of the cell. Can the intercellular $\mathrm{IP}_{3}$ waves occur in absence of $\mathrm{Ca}^{2+}$ waves or is the $\mathrm{Ca}^{2+}$ wave needed for the existence of the $\mathrm{IP}_{3}$ wave? If so, how are those chemical messengers influenced by the mechanical stimulation and what is the functional form of their cooperative response?

Finally, does one always see spatio-temporal downregulation in intercellular dynamics of $\mathrm{IP}_{3}$ following mechanical stimulation of a single cell in a confluent cell monolayer? Our model predicts that one should be able to see this transient downregulation followed by much slower recovery, but the experimental evidence of Hirose et al. (1999) so far is inconclusive on this matter. A separate experiment presented in Wallingford et al. (2002) gives partial support for our model prediction. They found that spontaneous $\mathrm{Ca}^{2+}$ waves in Xenopus died out after having travelled into $5-15$ cells around the initiation point; our model predicts that similar behaviour could also occur in epithelial cells. However, as the experiment of Wallingford et al. (2002) was different from the experiment of Hirose et al. (1999), one cannot directly translate the results between the two. Therefore, more experimental investigations are necessary to clarify this and other concerns raised in this paper.

\section{Acknowledgements}

The authors would like to thank Professor Hirose from the Department of Pharmacology, University of Tokyo, and Professor Vaughan-Jones from Department 
of Physiology, University of Oxford for their advice on calcium and $\mathrm{IP}_{3}$ signalling. This research was supported by EPSRC grant number GR/R68146/01.

\section{References}

Allbritton, N.L., Meyer, T., Stryer, L., 1992. Range of messenger action of calcium ion and inositol 1,4,5-trisphosphate. Science 258, 1812-1815.

Atri, A., Amundson, J., Clapham, D., Sneyd, J., 1993. A single-pool model for intracellular calcium oscillations and waves in the xenopus laevis oocyte. Biophys. J. 65, 1727-1739.

Berridge, M.J., Bootman, M.D., Lipp, P., 1998. Calcium-A life and death signal. Nature 395, 645-648.

Berridge, M.J., Lipp, P., Bootman, M.D., 2000. The versatility and universality of calcium signalling. Nat. Rev. Mol. Cell Biol. 1, 11-21.

Blackman, B.R., Thibault, L.E., Barbee, K.A. 2000. Selective modulation of endothelial cell $\left[\mathrm{Ca}^{2+}\right]_{i}$ response to flow by the onset rate of shear stress. J. Biomech. Eng. 122, 274-282.

Boitano, S., Dirksen, E.R., Sanderson, M.J., 1992. Intercellular propagation of calcium waves mediated by inositol trisphosphate. Science 258, 292-295.

Carafoli, E., 2003. The calcium-signalling saga: Tap water and protein crystals. Nat. Rev. Mol. Cell Biol. 4, 326-332.

Chicurel, M.E., Singer, R.H., Meyer, C.J., Ingber, D.E., 1998. Integrin binding and mechanical tension induce movement of mRNA and ribosomes to focal adhesions. Nature 392, 730-733.

Dolmetsch, R.E., Xu, K., Lewis, R.S., 1998. Calcium oscillations increase the efficiency and specificity of gene expression. Nature 392, 933-936.

Farge, E., 2003. Mechanical induction of twist in the Drosophila foregut/stomodeal primordium. Curr. Biol. 13, 1365-1377.

Felix, J.A., Woodruff, M.L., Dirksen, E.R., 1996. Strech increases inositol 1,4,5-trisphosphate concentration in airway epithelial cells. Am. J. Respir. Cell Mol. Biol. 14(3), 296-301.

Fink, C.C., Slepchenko, B., Moraru, I.I., Schaff, J., Watras, J., Loew, L.M., 1999. Morphological control of inositol-1,4,5-trisphosphate-dependent signals. J. Cell Biol. 147(5), 929-935.

Hirose, K., Kadowaki, S., Tanabe, M., Takeshima, H., Iino, M., 1999. Spatiotemporal dynamics of inositol 1,4,5-triphosphate that underlies complex $\mathrm{Ca}^{2+}$ mobilization patterns. Science 284, $1527-1530$.

Höfer, T., Venance, L., Giaume, C., 2002. Control and plasticity of intercellular calcium waves in astrocytes: A modeling approach. J. Neurosci. 22(12), 4850-4859.

Keener, J., Sneyd, J., 1998. Mathematical Physiology. Springer, Berlin Heidelberg New York.

Kummer, U., Olsen, L.F., Dixon, C.J., Green, A.K., Bornberg-Bauer, E., Baier, G., 2000. Switching from simple to complex oscillations in calcium signalling. Biophys. J. 79, 11881195.

Luzzi, V., Murtazina, D., Allbritton, N.L., 2000. Characterization of a biological detector cell for quantification of inositol 1,4,5-trisphosphate. Anal. Biochem. 277, 221-227.

Luzzi, V., Sims, C.E., Soughayer, J.S., Allbritton, N.L., 1998. The physiologic concentration of inostiol 1,4,5-trisphosphate in the oocytes of Xenopus laevis. J. Biol. Chem. 273(44), 2865728662.

Marks, A.R., 2003. Arrhythmias of the heart: Beyond ion channels. Nat. Med. 9(3), 263-264.

Meyer, T., Stryer, L., 1988. Molecular model for receptor-stimulated calcium spiking. Proc. Natl. Acad. Sci. USA 85, 5051-5055.

Meyer, T., Stryer, L., 1991. Calcium spiking. Annu. Rev. Biophys. Biophys. Chem. 20, 153-174.

Mishra, J., Bhalla, U.S., 2002. Simulations of inositol phosphate metabolism and its interaction with $\mathrm{InsP}_{3}$-mediated calcium release. Biophys. J. 83, 1298-1316.

Mohanti, M.J., Li, X., 2002. Strech-induced $\mathrm{Ca}^{2+}$ release via an $\mathrm{IP}_{3}$-insensitive $\mathrm{Ca}^{2+}$-channel. Am. J. Physiol. Cell Physiol. 283, 456-462.

Mohanti, M.J., Ye, M., Li, X., Rossi, N.F., 2001. Hypotonic swelling-induced $\mathrm{Ca}^{2+}$ release by an $\mathrm{IP}_{3}$-insensitive $\mathrm{Ca}^{2+}$ store. Am. J. Physiol. Cell Physiol. 281, C555-C562.

Murray, J.D., 1993. Mathematical Biology. Springer-Verlag, Berlin.

Okubo, Y., Kakizawa, S., Hirose, K., Iino, M., 2001. Visualization of $\mathrm{IP}_{3}$ dynamics reveals a novel AMPA receptor-triggered $\mathrm{IP}_{3}$ production pathway mediated by voltage-dependent $\mathrm{Ca}^{2+}$ influx in Purkinje cells. Neuron 32,113-122. 
Schuster, S., Marhl, M., Höfer, T., 2002. Modelling of simple and complex calcium oscillations: From single-cell responses to intercellular signalling. Eur. J. Biochem. 269, 1333-1355.

Sneyd, J., (Ed.), 2005. Tutorials in Mathematical Biosciences II Mathematical Modelling of Calcium Dynamics and Signal Transduction. Springer, Berlin Heidelberg New York.

Sneyd, J., Dale, P.D., Duffy, A., 1998a. Travelling waves in buffered systems: Applications to calcium waves. SIAM J. Appl. Math. 58(4), 1178-1192.

Sneyd, J., Wilkins, M., Strahonja, A., Sanderson, M.J., 1998b. Calcium waves and oscillations driven by an intercellular gradient of inositol $(1,4,5)$-trisphosphate. Biophys. Chem. 72, $101-$ 109.

Sneyd, J., Wetton, B.T.R., Charles, A.C., Sanderson, M.J., 1995. Intercellular calcium waves mediated by diffusion of inositol trisphosphate: A two-dimensional model. Am. J. Physiol. Cell Physiol. 37, 1537-1545.

Wallingford, J.B., Ewald, A.J., Harland, R.M., Fraser, S.E., 2002. Calcium signalling during convergent extension in Xenopus. Curr. Biol. 11, 652-661.

Wilkins, M., Sneyd, J., 1998. Intercellular spiral waves of calcium. J. Theor. Biol. 191, 299-308. 